\title{
Extension of L-Fuzzy Topological Tower Spaces
}

\author{
Hyei Kyung Lee \\ Department of Mathematics and Computer Science, RUCA University of Antwerp, Middleheimlaan \\ 1 B-2020 Antwerp, Belgium
}

\begin{abstract}
The purpose of this paper is to introduce the notions of $L$-fuzzy topological towers by using a completely distributive lattic $L$ and show that the category L-FPrTR of $L$-fuzzy pretopoplogical tower spaces and the category L-FPsTR of $L$-fuzzy pseudotopological tower spaces are extensional topological constructs. And we show that L-FPsTR is the cartesian closed topological extension of $\mathbf{L}$-FPrTR. Hence we show that $\mathbf{L}-\mathbf{F P s} T \mathbf{R}$ is a topological universe.
\end{abstract}

Key words : completely distributive lattice, $L$-fuzzy pseudotopological tower, $L$-fuzzy pretopological tower, cartesian closed, topological extension, topological universe.

\section{Introduction}

In [3], P. Eklund and W. Gahler defined the notions of generalized limit and Cauchy structures from the standpoint of general structure theory. Each of these structures is related to a certain covariant functor $\Phi=(\phi$, $\leqq$ ) from the category Set of sets to the category SLat of semilattices satisfying three conditions $(\mathrm{P}),(\mathrm{Pr})$ and (D) respectively concerning the existence, preservation and distributivity of $\Phi$-products which generalize the usual notions of filter products. Under the assumption that $\phi$ is connected i.e. $\phi(1)$ (with $1=\{0\}$ ) is a singleton set which gives a natural transformation $\eta: i d \rightarrow \phi$, they defined the notions of $\Phi$-limit spaces and showed the category $\Phi$-Lim of $\Phi$-limit spaces is cartesian closed. And in [10], K. C. Min and Y. J. Lee introduced the notions of $L$-prefilter (In this paper, it is called $L$

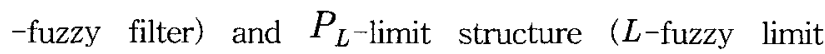
structure) generalizing the neighborhood system of points in $L$-fuzzy topological space in the scheme of $\Phi$-limit structure in [10].

On the other hand probabilistic convergence spaces were defined in 1989 by Florescu [4], in terms of net convergence. The filter formulation considered was introduced by G. D. Richardson and D. C. Kent [13]. The fundamental idea is to assign a probability to the convergence of a given filter to a given point. In 1997, P. Brock and D. C. Kent [2], introduced the limit tower spaces whose objects resemble probabilistic convergence spaces.

접수일자 : 2004년 12월 16일

완료일자 : 2005년 2월 18일

"This Paper was supported by Dr. Lee Sewoong's

Studying Fund, 2002."
In this paper, we introduce the notions of $L$-fuzzy limit tower, $L$-fuzzy pseudotopological tower and $L$ -fuzzy pretopological tower. And show that the category L-FLTR of $L$-fuzzy limit tower spaces and $L$-fuzzy continuous maps is a cartesian closed topological construct and the category L-FPsTR of $L$-fuzzy pseudotopological tower spaces and the category L-FPrTR of $L$-fuzzy pretopological tower spaces are extensional topological construct and the category L-FPsTR is the cartesian closed topological extension of the category L-FPrTR. Hence L-FPsTR is the topological universe extension of L-FPrTR.

\section{Preliminaries}

Throughout this paper, we let $L$ be a completely distributive complete lattice with different least and last elements denoted by $0_{L}, 1_{L}$, respectively. Recall that the complete distributive law $\vee\left(\alpha_{i} \wedge \alpha\right)=\left(\vee \cdot \alpha_{i}\right) \wedge \alpha$ holds and with an order reversing involution $x \rightarrow x^{\prime}$ ( $x \in L$ ). If $X$ is any set, the map $f: X \rightarrow L$ is called an $L$-fuzzy subset of $X$.

$L^{X}$ denotes the set of all $L$-fuzzy subsets of $X$.

Let $X$ be a set. A non-empty subset $F$ of $L^{X}$ is called an $L$-fuzzy filter on $X$ if

(LF1) if $A \in F$ and $A \subseteq B$, then $B \in F$,

(LF2) for all $A, B \in F$, we have $A \cap B \in F$,

(LF3) $\underline{\alpha} \in F, \forall \alpha>0_{L}$ where $\underline{0_{L}} \notin F$.

For a set $X$, let $F_{L}(X)$ be the set of all $L$-fuzzy filters on $X$. 
For any set $X, Y$ and a map $f: X \rightarrow Y$ and the map $F_{L}(f(F)): L^{Y} \rightarrow\{0,1\}$ defined by $F_{L}(f(F))(g)=F(g \circ f) \quad \forall g \in L^{Y}$ is an $L$-fuzzy filter on $Y$ and we write $F_{L}(f(F))$ simply by $f(F)$.

A subset $\mathrm{B}$ of an $L$-fuzzy filter $F$ is called a base for $F$ if $\forall A \in F, \exists B \in \mathbf{B}$ such that $B \leqq A$. For a given set $X$, a subset $\mathrm{B}$ of $L^{X}$ is called an $L$-fuzzy filter base on $X$ if it satisfies the following :

(LFB1) $\underline{\alpha} \in \mathbf{B}, \forall \alpha>0_{L}$ and $\underline{0_{L}} \notin \mathbf{B}$,

(LFB2) $\forall A, B \in \mathrm{B}, \exists C \in \mathrm{B}$ s.t $C \leqq A \wedge B$.

Definition 2.1. An $L$-fuzzy filter $U$ on $X$ is an $u l t r a L$ -fuzzy filter if there is no other $L$-fuzzy filter finer than $U$.

Definition 2.2.[10] An L-fuzzy limit structure $t$ is a subset of $F_{L}(X) \times X$, subject to the following axioms : where $F \in t(x)$ means $(F, x) \in t$.

$\left(\right.$ LFL1) $<x>=\left\{A \in L^{X}: \mu_{A}(x)>0_{L}\right\} \in t(x)$, for all $x \in X$,

(LFL2) if $F \in t(x)$ and $F \subseteq G$ then $G \in t(x)$, (LFL3) if $F, G \in t(x)$, then $F \cap G \in t(x)$.

The pair $(X, t)$ is called an $L$-fuzzy limit space. If $F \in t(x)$, then we say that $F$ converges to $x$ and $x$ is a limit point of $F$.

Definition 2.3. A map $f:\left(X, t_{X}\right) \rightarrow\left(Y, t_{Y}\right)$ between $L$ -fuzzy limit spaces is said to be $L$-fuzzy continuous if $F \in t_{X}(x)$, then $f(F) \in t_{Y}(f(x))$.

Let $\mathrm{L}$-FLim denote the category of all $L$-fuzzy limit spaces and all $L$-fuzzy continuous maps.

Theorem 2.4.[10] The category L-FLim is a topological construct.

Theorem 2.5.[10] The category L-FLim is a cartesian closed category.

Theorem 2.6.[10] The category L-FLim is a quasitopos.

\section{L-fuzzy limit tower spaces}

Definition 3.1. An $L$-fuzzy limit tower $\bar{t}$ is a family $\left\{t_{\alpha}: 0 \leqq \alpha \leqq \infty\right\}$ of $L$-fuzzy limit structures such that :

(LFTR1) $t_{\alpha}$ is an $L$-fuzzy limit structure, for each $\alpha \in[0, \infty]$,

(LFTR2) $t_{\infty}$ is the indiscrete topology,

(LFTR3) $t_{\alpha}=\cap_{\beta>\alpha} t_{\hat{\beta}}$, for each $\alpha \in[0, \infty)$.

The pair $(X, \bar{t})$ is called an $L$-fuzzy limit tower space.

Definition 3.2. For any $L$-fuzzy limit tower spaces $\left(X, \overline{t_{X}}\right)$ and $\left(Y, \overline{t_{Y}}\right)$, a map $f:\left(X, \overline{t_{X}}\right) \rightarrow\left(Y, \overline{t_{Y}}\right)$ is L-fuzzy continuous if $F \in t_{\alpha}^{X}(x)$ then $f(F) \in t_{\alpha}^{Y}(f(x))$ for each $0 \leqq \alpha \leqq \infty$ where $\overline{t_{X}}$ $=\left(t_{\alpha}^{X}\right), \overline{t_{Y}}=\left(t_{\alpha}^{Y}\right)$.

Let $\mathbf{L}$-FLTR denote the category of all $L$-fuzzy limit tower spaces and all $L$-fuzzy continuous maps.

Theorem 3.3. The category L-FLTR is topological category.

Proof. To show that L-FLTR is initially complete, let $\left(f_{i}: X \rightarrow\left(Y_{i}, \bar{t}_{i}\right)\right)_{i \in l}$, be a source such that $\left(Y_{i}, \bar{t}_{i}\right)$ is an $L$-fuzzy limit tower space for each $i \in I$. We define $\dot{t}=$ $\left(t_{\alpha}\right)$ by $F \in t_{\alpha}(x)$ iff $f_{i}(F) \in t_{\alpha}^{i}(f(x))$ for all $i \in I$, $x \in X$ and for each $\alpha \in[0, \infty)$. And $t_{\infty}(x)=F(X)$. Then $\bar{t}$ is the initial $L$-fuzzy limit tower on $X$ with respect to $\left(f_{i}\right)_{i \in I}$ and the remainder is trivial.

\section{Function spaces}

Let $\left(X, \overline{t_{X}}\right)$ and $\left(Y, \bar{t}_{Y}\right)$ be $L$-fuzzy limit tower spaces and let $C(X, Y)$ be the set of all $L$-fuzzy continuous maps from $X$ into $Y$. Let $H$ be a prefilter on $C(X, Y)$ and $F$ be an $L$-fuzzy filter on $X$. For $H \in H$ and $\mathrm{F} \in F$, define an $L$-fuzzy set $\mathrm{H}(\mathrm{F}): Y \rightarrow L$ by

$\mu_{H(F)}(y)$

$=\left\{\begin{array}{lr}\sup _{(x, g) \in e v^{-1}(y)} \mu_{H(g)} \wedge \mu_{F}(x) \text { if } e v^{-1}(y) \neq \phi \\ 0 & \text { otherwise }\end{array}\right.$

where $e v: X \times C(X, Y) \rightarrow Y$ is the evaluation map. We note that if $\mathrm{H}_{1} \subseteq \mathrm{H}_{2}$ in $H$ and $\mathrm{F}_{1} \subseteq \mathrm{F}_{2}$ in $F$, then $\mathrm{H}_{1}$ $\left(\mathrm{F}_{1}\right) \subseteq \mathrm{H}_{2}\left(\mathrm{~F}_{2}\right)$. Thus $\mathbf{H}(\mathbf{F})=\left\{B \in L^{Y}: H(F) \subseteq B\right.$, for some $H \in \mathbf{H}, F \in \mathbf{F}\}$ is an $L$-fuzzy filter on $Y$. Define a $\bar{t}=\left(t_{\alpha}\right)$ as follows : for each $f \in C(X, Y)$, $H \in t_{\alpha}(f)$ if and only if for any $x \in X$, if $F \in t_{\alpha}^{X}(x)$ 
then $H(F) \in t_{\alpha}^{Y}(f(x))$ for each $\alpha \in[0, \infty)$. And $t_{\infty}=F_{L}(C(X, Y))$.

Proposition 3.4. For objects $\left(X, \bar{t}_{X}\right),\left(Y, \bar{t}_{Y}\right)$ in L-FLTR, the L-fuzzy limit tower $\bar{t}$ on $C(X, Y)$ is an L-fuzzy limit tower.

Proof. Let $f \in C(X, Y), H \in F(C(X, Y))$.

(LFTR1): $\bar{t}=\left(t_{\alpha}\right)_{\alpha}, 0 \leqq \alpha \leqq \infty$ is an $L$-fuzzy limit structure.

(LFL1) Let $F \in t_{\alpha}^{X}$. Then $f(F) \subseteq<f>(F)$ and since $f$ is L-fuzzy continuous, $f(F) \in t_{\alpha}^{Y}(f(x))$, $<f>(F) \in t_{\alpha}^{Y}(f(x))$. By definition of $t_{\alpha}$, $<f>\in t_{\alpha}(f)$.

(LFL2) Suppose $H \in t_{\alpha}(f)$ and $H \subseteq K$. Then for $F \in t_{\alpha}^{X}(x), \quad H(F) \in t_{\alpha}^{Y}(f(x))$ and $H(F) \subseteq K(F)$ Thus $K(F) \in t_{\alpha}^{Y}(f(x))$. Hence $K \in t_{\alpha}(f)$.

(LFL3) Suppose $H, K \in t_{\alpha}(f)$. Then for $F \in t_{\alpha}^{X}(x)$, $H(F), K(F) \in t_{\alpha}^{Y}(f(x)) . \quad H(F) \cap K(F) \in t_{\alpha}^{Y}(f(x))$. But $(H \cap K)(F)=H(F) \cap K(F)$. So $(H \cap K)(F)$ $\in t_{\alpha}^{Y}(f(x))$. Thus $H \cap K \in t_{\alpha}(f)$.

(LFTR2): It is given by definition of $\dot{t}$ on $C(X, Y)$.

(LFTR3): To show that $t_{\alpha}=\bigcap_{\beta>\alpha} t_{\beta}$, let $H \in t_{\alpha}(f)$ then for $F \in t_{\alpha}^{X}(x), H(F) \in t_{\alpha}^{Y}(f(x))$. Since $\overline{t_{Y}}$ is an $L$ -fuzzy limit tower, for all $\beta>\alpha, H(F) \in \cap t_{\beta}^{Y}(f(x))$. So $H \in \cap t_{\beta}(f)$ for all from $\beta>\alpha$. Conversely let $H \in \cap t_{\beta}(f)$ for all $\beta>\alpha$ then for $F \in \cap t_{\beta}^{X}(x)$, $H(F) \in \cap t_{\beta}^{Y}(f(x))$ and hence $H(F) \in t_{\alpha}^{Y}(f(x))$. Thus $H \in t_{\alpha}(f)$.

The space $C(X, Y)$ equipped with $L$-fuzzy limit tower is denoted as $[X, Y]$.

Theorem 3.5. The evaluation map ev $X \times[X, Y] \rightarrow Y$ is $L-f u z z y$ continuous.

Proof. Let $F \rightarrow(x, f)$ in $\left(X, \bar{t}_{X}\right) \times(C(X, Y), \bar{t})$ then for each $\alpha, \operatorname{Pr}_{2}(F) \in t_{\alpha}(f)$ in $C(X, Y), \operatorname{Pr}_{1}(F) \in t_{\alpha}^{X}(x)$ in $\mathrm{X}, \operatorname{Pr}_{2}(F)\left(\operatorname{Pr}_{1}(F)\right) \in t_{\alpha}^{Y}(f(x))$ in $\mathrm{Y}$. Thus it is enough to show that $\operatorname{Pr}_{2}(F)\left(\operatorname{Pr}_{1}(F)\right) \subseteq e v(F)$. Take any $T \in P r_{2}(F)\left(P r_{1}(F)\right)$. Then there exist $K \in P r_{2}(F)$ and $L \in \operatorname{Pr}_{1}(F)$ with $K(L) \subseteq T$. Take any $A, B \in F$ such that $\operatorname{Pr}_{2}(A) \subseteq K$ and $\operatorname{Pr}_{1}(B) \subseteq L$. Let $C=A \cap B$. Then $C \in F$ and $e v(C) \subseteq P r_{2}(C)\left(P r_{1}(C)\right)$, by routine work. Hence $e v(C) \subseteq P r_{2}(B)\left(P r_{1}(A)\right) \subseteq K(L) \subseteq T$.
Theorem 3.6. Let $\left(X, \bar{t}_{X}\right), \quad\left(Y, \bar{t}_{Y}\right), \quad\left(Z, \bar{t}_{Z}\right) \in$ [L-FLTR| and $f: X \times Z \rightarrow Y$ be L-fuzzy continuous. Then there exists a unique fuzzy continuous map $f^{*}: Z \rightarrow C(X, Y)$ such that $e v \circ\left(i d \times f^{*}\right)=f$.

Proof. Define a map $f^{*}: Z \rightarrow C(X, Y)$ by $f^{*}(z)(x)=f(x, z)(z \in Z, x \in X)$. Then we can show that this map is unique $L$-fuzzy continuous, where $[z]: X \rightarrow Z$ is a constant map with value $z$.

To show that $f^{*}$ is $L$-fuzzy continuous, let $G \in t_{\alpha}^{Z}(z)$ and $F \in t_{\alpha}^{X}(x)$. Then for each $F \in F, G \in G$, define an $L$-fuzzy set $\quad[\mathrm{F}, \quad \mathrm{G}]: X \times Z \rightarrow L \quad$ by $\mu_{[F, G]}(x, z)=\mu_{F}(x) \wedge \mu_{G}(z)$ and $\mathrm{B}=\{[\mathrm{F}, \mathrm{G}]: \mathrm{F} \in F, \mathrm{G}$ $\in G$ \}. Then this forms a basis for an $L$-fuzzy filter $V$ on $X \times Z$. Since $f$ is $L$-fuzzy continuous, $f(V) \in t_{\alpha}^{Y}(f(x))$ in $Y$. Thus it is enough to show that $f^{*}(G)(F)=f([F, G]) \quad: \quad$ Suppose $e v^{-1}(y) \neq \varnothing \quad$ and $S=\left\{g \in C(X, Y):(x, y) \in e v^{-1}(y)\right.$ for some $\left.x \in X, f^{*-1}(y) \neq \varnothing\right\} \neq \varnothing$. Then $f^{-1}(y) \neq \varnothing$ and

$$
\begin{aligned}
& \mu_{f^{*}(G)(F)}(y) \\
& =\sup _{(x, y) \in e v^{-1}(y)} \mu_{f^{*}(G)}(g) \wedge \mu_{F}(x) \\
& =\sup _{(x, z) \in e v^{-1}(y)}\left(\sup _{z \in f^{*-1}(y)} \mu_{G}(z)\right) \wedge \mu_{F}(x) \\
& =\sup _{(x, z) \in e v^{-1}(y)} s u p_{z \in f^{*-1}(y)}\left(\mu_{G}(z) \wedge \mu_{F}(x)\right) \\
& =\sup _{(x, z) \in f^{-1}(y)} \mu_{G}(z) \wedge \mu_{F}(x) \\
& =\mu_{f([F, G])}(y) .
\end{aligned}
$$

If $e v^{-1}(y)=\varnothing \quad$ or $S=\varnothing$, then $f^{-1}(y)=\varnothing \quad$ and $\mu_{f(G)}(F)(y)=\mu_{f([F, G])}(y)=0$. So $f^{*}(G) \in t_{\alpha}\left(f^{*}(z)\right)$. Obviously ev $\circ\left(i d \times f^{*}\right)=f$ and a map $f^{*}$ is a unique.

\section{Categorical viewpoints}

A category $\mathbf{A}$ is said to be cartesian closed [5] if (1) for any pair $\mathrm{A}, \mathrm{B}$ of objects there exists a product $\mathrm{A} \times \mathrm{B}$, and (2) for any object $\mathrm{A}$, the functor $\mathrm{A}^{\times} \ldots: A \rightarrow A$ has a right adjoint. Therefore by above Proposition 3.4., Theorem 3.5. and Theorem 3.6., we obtain :

Theorem 3.7. The category L-FLTR is a cartesian closed topological category.

Throughout this paper, we refer to [5] for category theory.

\section{L-fuzzy pretopological tower spaces}

Definition 4.1. An $L$-fuzzy limit tower is called a $L$-fuzzy pretopological tower on $X$ if for an $L$-fuzzy 
limit tower $\bar{t}=\left(t_{\alpha}\right), 0 \leqq \alpha \leqq \infty$ and for all $F \in t_{\alpha}(x)$, $\cap F \in t_{\alpha}(x)$ for any $x \in X$.

The pair $(X, \bar{t})$ is called an $L$-fuzzy pretopological tower space.

Let L-FPrTR be the category of all $L$-fuzzy pretopological tower spaces and all $L$-fuzzy continuous maps.

Proposition 4.2. The category L-FPrTR is a topological construct.

Proof. The initial structures in L-FPrTR can be described in the same way as those in L-FLTR.

Proposition 4.3. The category L-FLTR, and L-FPrTR are well-fibred topological constructs and L-FPrTR is initially closed in L-FLTR, hence bireflective.

Definition 4.4. [6]. Let $\mathbf{A}$ be a well-fibred topological construct:

(1) A partial morphism from $X$ to $Y$ is a morphism $f: Z \rightarrow Y$ whose domain $Z$ is a subspace of $X$.

(2) Partial morphism to $Y$ are representable provided $Y$ can be embedded via the addition of a single point $p$ into an object $Y^{\#}$ with the property that for every partial morphism $f: Z \rightarrow Y$, the map $f^{X}: X \rightarrow Y^{\sharp} \quad$ defined by $f^{X}(x)=f(x) \quad$ if $x \in Z, f^{X}(x)=p$ if $x \notin Z$ is a morphism.

The object $Y^{\#}$ is called the one point extension of $Y$.

(3) $\mathbf{A}$ is called extensional if partial morphisms into all $\mathbf{A}$-objects are representable.

\section{Theorem 4.5. L-FPrTR is extensional.}

Proof. Suppose $\left(X, \overline{t_{X}}\right)$ is an object in L-FPrTR. Let $X^{\#}=X \cup\{p\}, p \notin X$ and define $\overline{t_{\#}}$ on $X^{\#}$ as follows that for all $x \in X^{\#}$ and $F \in F_{L}\left(X^{\#}\right)$, if $x=p$ or $F=\langle p\rangle$ then $F \in t_{0}^{\ddagger}(x) \quad$ and $\quad$ if $x \neq p, F \neq<p>, 0 \leqq \alpha<\infty$ then $F \in t_{\alpha}^{\#}(x) \quad$ if and only if $\left.F\right|_{X} \in t_{\alpha}^{X}(x)$. And $t_{\infty}^{\#}(x)=F_{L}(X)$. Then (1) $\left(X^{\#}, \overline{t^{\#}}\right)$ is an object in L-FPrTR. Clearly $\left(\overline{t_{\alpha}^{\#}}\right)_{\alpha}$ is an $L$-fuzzy limit tower. Enough to show that it is $L$ -fuzzy pretopological. For all $F \in t_{\alpha}^{\#}(x)$, if $x=p$ or $F=\langle p\rangle$ then $\cap F \in t_{0}^{\#}(x) \subseteq t_{\alpha}^{\#}(x)$ and if $x \neq p, F \neq<p>$ then $\left.F\right|_{X} \in t_{\alpha}^{X}(x)$ and since $t_{\alpha}^{X}$ is an $L$-fuzzy pretopological tower and $\left.\{\cap F\}\right|_{X}=\cap\left\{\left.F\right|_{X}\right\}$,
$\cap\left\{\left.F\right|_{X}\right\} \in t_{\alpha}^{X}(x)$. Thus $\cap F \in t_{\alpha}^{\#}(x)$. (2) The inclusion $i: X \rightarrow X^{\sharp}$ is initial because any $L$-fuzzy filter $F$ on $X$ and $x \in X,\left.i(F)\right|_{X}=F$, and so $F \in t_{\alpha}^{X}(x)$ if and only if $i(F) \in t_{\alpha}^{\#}(i(x))$. (3) For every partial morphism from $f: A \rightarrow X, A \subseteq Y$, the function $f^{Y}: Y \rightarrow X^{\#}$ defined by $f^{Y}(y)=f(y)$ if $y \in A$ and $f^{Y}(y)=p$ if $y \notin A$ then it is obviously $L$-fuzzy continuous. Therefore $\mathbf{L}-\mathbf{F P r T R}$ is extensional.

\section{L-fuzzy pseudotopological tower spaces}

By weakening the axioms of convergence in an $L$ -fuzzy pretopological tower spaces, we will obtain a cartesian closed topological extension of L-FPrTR.

Definition 5.1. An $L$-fuzzy limit tower is called an $L$-fuzzy pseudotopological tower on $X$ if for $L$-fuzzy limit tower $\bar{t}=\left(t_{\alpha}\right), F \in t_{\alpha}(x)$ if and only if every ultra $L$-fuzzy filter $U$ finer than $F, U \in t_{\alpha}(x)$ for any $x \in X$, for each $\alpha \in[0, \infty]$.

The pair $(X, \dot{t})$ is called an $L$-fuzzy pseudotopological tower space.

Let L-FPsTR be the category of all $L$-fuzzy pseudotopological tower spaces and all $L$-fuzzy continuous maps.

Remark 5.2. Since every $L$-fuzzy filter is the intersection of all finer ultra $L$-fuzzy filters, $\mathbf{L}$-FPrTR is a subcategory of L-FPsTR.

Proposition 5.3. The category L-FPsTR is a topological construct.

Proof. The initial structures in $\mathbf{L}$-FPrTR can be described in the same way as those in L-FPsTR.

Proposition 5.4. The category $\mathbf{L}-\mathbf{F P r T R}$ is initially closed in L-FPsTR, hence L-FPrTR is a bireflective subcategory of L-FPsTR.

Proposition 5.5. For objects $\left(X, \overline{t_{X}}\right),\left(Y, \overline{t_{Y}}\right)$ in L-FPsTR, the L-fuzzy limit tower $\bar{t}$ on $C(X, Y)$ is an L-fuzzy pseudotopological tower.

Proof. We enough to show that this $L$-fuzzy limit tower $t$ on $C(X, Y)$ is pseudotopological.: if $K$ is a ultra $L$ -fuzzy filter containing $H$ such that $K \in t_{\alpha}^{H}(f)$ then for each $F \in t_{\alpha}^{X}(x), \quad K(F) \in t_{\alpha}^{Y}(f(x))$ and since 
$\left(Y, \bar{t}_{Y}\right) \in\lfloor\mathbf{L}-$ FPsTR $\mid \quad$ and $\quad H(F) \subseteq K(F)$, $H(F) \in t_{\alpha}^{Y}(f(x))$. Thus $H \in t_{\alpha}(f)$.

As a corollary of Theorem 3.7., we have

Theorem 5.6. The evaluation map ev $: X \times[X, Y] \rightarrow Y$ is $L$-fuzzy continuous.

Theorem 5.7. Let $\left(X, \overline{t_{X}}\right), \quad\left(Y, \overline{t_{Y}}\right), \quad\left(Z, \bar{t}_{Z}\right) \in$ IL-FPsTR| and $f: X \times Z \rightarrow Y$ be $L$-fuzzy continuous. Then there exists a unique fuzzy continuous map $f^{*}: Z \rightarrow C(X, Y)$ such that $e v \circ\left(i d \times f^{*}\right)=f$.

Theorem 5.8. L-FPsTR is a cartesian closed topological category.

By the above theorem, $\mathbf{L}$-FPsTR is a cartesian closed topological extension of $\mathrm{L}-$ FPrTR.

\section{Proposition 5.9. L-FPsTR is extensional.}

Proof. Suppose $\left(\mathrm{X}, \bar{t}_{X}\right)$ is an object in L-FPsTR. Let $X^{\#}=X \cup\{p\}, p \notin X$ and define $\overline{t^{\#}}=\left(t_{\alpha}^{\#}\right)$ on $X^{\#}$ as follows : if $x=p$ or $F=<p>$ then for $F \in F_{L}\left(X^{\#}\right)$, $F \in t_{0}^{\#}(x)$ and if $x \neq p, F \neq<p>$ then for $F \in F_{L}\left(X^{\#}\right), F \in t_{\alpha}^{\#}(x)$ if and only if $\left.F\right|_{X} \in t_{\alpha}^{X}(x)$ for each $\alpha \in[0, \infty)$. And $t_{\infty}^{\#}=F_{L}\left(X^{\#}\right)$. Using the Theorem 4.5 , it is sufficient to show that $\overline{t^{\#}}=\left(t_{\alpha}^{\#}\right)$ is $L$-fuzzy pseudotopological. Let $F \in F_{L}\left(X^{\#}\right)$ and for every ultra $L$-fuzzy filter $U \supset F, U \in t_{\alpha}^{\#}(x)$. Then if $x=p$ or $F=\langle p\rangle$ then by definition of $\overline{t^{\#}}$, $F \in t_{0}(x) \subset t_{\alpha}^{\#}(x)$, for all $\alpha \in[0, \infty]$. Otherwise i.e., if $x \neq p, F \neq<p>$ then $\left.F\right|_{x}$ is a proper $L$-fuzzy filter on $X$. Since $j\left(\left.F\right|_{X}\right) \supset F$, every ultra $L$-fuzzy filter $V$ on $X$ with $\left.V \supset F\right|_{X}$ can be represented as $\left.U\right|_{X}$ of some ultra $L$-fuzzy filter $U \supset F$ with $U \neq<<p>$ : from $U \in t_{\alpha}^{\#}(x), V=\left.U\right|_{X} \in t_{\alpha}^{X}(x)$. Since $\overline{t_{X}}=\left(t_{\alpha}^{X}\right)$ is an $L$ -fuzzy pseudo topological tower, $\left.F\right|_{X} \in t_{\alpha}^{X}(x)$. Thus $F \in t_{\alpha}^{\#}(x)$. Therefore L-FPsTR is extensional.

Definition 5.10.[11] A well fibred topological construct is called a topological quasitopos or a topological universe if it is both cartesiian closed and extensional.

By Theorem 5.8. and Theorem 5.9., we have

Theorem 5.11. L-FPsTR is a topological universe.

Question : We proved L-FPsTR is cartesian closed extensional topological construct of $\mathbf{L}$-FPrTR. Hence
L-FPsTR is topological universe of $\mathbf{L}$-FPrTR. So now we have a question that is L-FPsTR the "smallest" topological universe extension of L-FPrTR ?' In topological case [7] it was already proved i.e., the category PsTop of pseudotopological spaces and continuous maps is topological universe of the category PrTop of pretopological spaces and continuous maps. Moreover it was proved that PsTop is the topological universe hull of the construct PrTop. Thus I guess this answer is "yes". But there are some problems to prove it. First of all, we should obtain $L$-fuzzy topological tower $\dot{t}$ on $[0, \infty]$ which plays a similar role in $L$-fuzzy topological tower space as the Sierpinski space 2 in topological space. We will investigate the topological universe hull of L-FPrTR.

\section{References}

[1] J. Adamek, H. Herrlich and G. E. Strecker, Abstract and Concrete Categories, John Wiley and Sons, Inc. 1990.

[2] P. Brock and D. C. Kent, Approach spaces, limit tower spaces and probabilistic convergence spaces, Applied Categorical Structures 5 (1997), 99-110.

[3] P. Eklind and Gahler, Contributing to Fuzzy Convergence, in: Recent Developments of General Topology and its Applications, international Conference in Memory of Felix Hausdorff Math Research 67, Berlin (1992).

[4] L. C. Florescu, Probabilistic convergence structures, Aequatioines Math 38 (1989), 123-145.

[5] H. Herrich ,Cartesian closed topological categories, Math Colloq. Univ. Cap Town 9 (1974), 1-16.

[6] H. Herrich, Hereditary topological constructs, in $Z$. Frolik (ed.), General Topology and its Relations to Modern Analysis and Algebra VI, Proc. Sixth Prague Topological Symposium, Heldermann Verlag, Berlin, (1988), 249-262.

[7] E. Lowen-Colebunders, F. Schwarz, Improving Top: PrTop and PsTop, Ctegory Theory at Work, Heldermann Verlag, Berlin, (1991), 21-34.

[8] H. Herrich, L .D .Nel, Cartesian closed topological hulls, Proc. Amer. Math Soc. 62 (1977), 215-222.

[9] E. Lowen, Function classes of Cauchy continuous maps, Pure and applied mathematics 123 Macel Dekker, Inc. (New York, 1989).

[10] K. C. Min, Y. J. Lee, $P_{L^{-}}$limit structure in $\mathrm{L}$-fuzzy topology, to appear.

[11] L, D. Nel, Topological universes and smooth Gelfand-Naimark duality, Contemporary Math 30 (1984), 244-276.

[12] G. Preuss, Theory of topological structures, Kluwer Academic Publishers, 1987. 
퍼지 및 지능시스템학회 논문지 2005 , Vol. 15 , No. 3

[13] G. D. Richardson and D. C. Kent, Probabilistic convergence spaces, J. Austrl. Math Soc (Series A) 61 (1996), 400-420.

\section{저 자 소 개}

\section{Hyei Kyung Lee}

She recirved her Ph.D in Mathematics from Univ.of Yonsei in Korea. She served as a Research Fellow from 2002 to 2004 at the Univ. of Antwerp, Belgium in the research group Analytical and Topological Structures.

During the period she has worked on optimization in fuzzy control and has worked on countability properties in approach theory. Her major interest is fuzzy topology, category theory, approach theory and fuzzy control. 